\title{
Kerr black holes as retro-MACHOs
}

\author{
F. De Paolis ${ }^{1}$, A. Geralico ${ }^{1,2}$, G. Ingrosso ${ }^{1}$, A. A. Nucita ${ }^{1}$, and A. Qadir ${ }^{3,4}$ \\ 1 Dipartimento di Fisica, Università di Lecce, and INFN, Sezione di Lecce, via Arnesano, CP 193, 73100 Lecce, Italy \\ 2 International Center for Relativistic Astrophysics - ICRA, University of Rome "La Sapienza", 00185 Roma, Italy \\ 3 Department of Mathematics, Quaid-i-Azam University, Islamabad, Pakistan \\ ${ }^{4}$ Department of Mathematical Sciences, King Fahd university of Petroleum and Minerals, Dhahran 31261, Saudi Arabia
}

Received 14 May 2003 / Accepted 17 October 2003

\begin{abstract}
Gravitational lensing is a well known phenomenon predicted by the General Theory of Relativity. It is now a welldeveloped observational technique in astronomy and is considered to be a fundamental tool for acquiring information about the nature and distribution of dark matter. In particular, gravitational lensing experiments may be used to search for black holes. It has been proposed that a Schwarzschild black hole may act as a retro-lens (Holz \& Wheeler 2002) which, if illuminated by a powerful light source (e.g. the Sun), deflects light ray paths to large bending angles so that the light may reach the observer. Here, by considering the strong field limit in the deflection angle and confining our analysis to the black hole equatorial plane, we extend the Holz-Wheeler results to slowly spinning Kerr black holes. By considering the Holz-Wheeler geometrical configuration for the lens, source and observer we find that the inclusion of rotation does not substantially change the brightness of the retro-lensing images with respect to the Schwarzschild case. We also discuss the possibility that the next generation space-based telescopes may detect such retro-images and eventually put limits on the rotational parameter of the black hole.
\end{abstract}

Key words. gravitation - gravitational lensing

\section{Introduction}

Gravitational lensing of electromagnetic waves is a wellunderstood phenomenon (see e.g. Schneider et al. 1992 for a comprehensive treatment) predicted by the theory of General Relativity. In the last years, gravitational lensing has been also used as an observational tool to investigate the mass distribution both in galaxy clusters (using distant QSOs as sources, Canizares 1982) and in galactic halos. In the latter case, the phenomenon, which is called gravitational microlensing, led to the discovery of Massive Astrophysical Compact Halo Objects (MACHOs) in the halo of our Galaxy (Alcock et al. 1993; Aubourg et al. 1993).

As a dark object moves across the source-observer line of sight it acts as a gravitational lens leading to the formation of two unresolved images of the source and to an overall source light magnification. Despite the low probability of observing such events, Paczyńsky (1986) showed that the continuous observation of at least $\simeq 10^{6}$ stars implies a non-negligible chance of detecting a microlensing event towards the LMC.

Since the early 1990s several collaborations (MACHO (see e.g. Alcock et al. 1993), EROS (Aubourg et al. 1993) and OGLE (Stanek et al. 1995)) have been monitoring millions of stars towards the LMC, the SMC, as well as towards the

Send offprint requests to: F. De Paolis, e-mail: Francesco.DePaolis@le.infn.it galactic center detecting hundreds of microlensing events due to MACHOs along the line of sight. Unfortunately, the physical nature of MACHOs is still unknown, since the observed light curves of a microlensing event can be reproduced by models depending on a number of free parameters (mass, distance and transverse velocity of the lens). So, different populations of objects such as brown dwarfs, white dwarfs, main sequence stars and black holes may be consistent with microlensing observations. In particular, it is generally accepted that a population of black holes may exist in the Galaxy and it has been claimed that these compact objects have already been detected in microlensing surveys (Quinn et al. 1999; Bennett et al. 2002; Agol et al. 2002). At least 6 extremely long events detected by the MACHO, GMAN and MPS collaborations towards the galactic bulge (Bennett et al. 2002) exhibit very strong microlensing parallax signals which lead to mass estimates up to $\simeq 10 M_{\odot}$. Since the estimated upper limits on the absolute lens brightness are always less than $\sim 1 L_{\odot}$, this favors the stellar black hole hypothesis (Mao et al. 2002) and suggests that a substantial fraction of the galactic lenses may be massive stellar remnants.

Despite some experimental uncertainties, the theory of gravitational lensing, which was originally developed in the weak field approximation, has successfully explained all gravitational lensing observations from the giant blue luminous arcs in the rich cluster of galaxies (Lynds \& Petrosian 1989; Soucail et al. 1987) to the first Einstein ring MG 1131+0456 at redshift 
$z \simeq 1.13$ (Hewitt et al. 1988) to microlensing events detected towards the SMC, LMC, the galactic bulge and the M 31 galaxy (see e.g. Alcock et al. 2000).

Moreover, by using the next generation of high resolution imaging telescopes, it would be possible to test the theory of gravitational lensing in the presence of strong gravitational fields since the inspection of the images formed will allow us to investigate regions very close to the surface of compact massive objects such as neutron stars and black holes.

In a very interesting paper Holz \& Wheeler (2002) proposed that a Schwarzschild black hole may act as retro-lens or retro-MACHO. This means that if the black hole is illuminated by a powerful light source, photons, due to its strong gravitational field, can be deflected by large bending angles. In the case of perfect alignment of source, black hole and Earth (with the Earth in the middle), the bending angles are odd multiples of $\pi$ leading to a series of circular rings.

However, black holes would be characterized by a non-zero intrinsic angular momentum, which breaks the Schwarzschild spherical symmetry and affects the gravitational field around the compact object. Thus, we expect a modification in the phenomenology of the gravitational lensing. Here, we investigate the retro-lensing phenomenon for slowly rotating Kerr black holes considering photon trajectories close to the black hole equatorial plane. Our treatment follows the second order expansion (given by Bozza 2003) of the large deflection angles of light rays winding around the retro-MACHO in the impact parameter $b$.

This paper is structured as follows. In Sect. 2 we briefly review the Schwarzschild retro-MACHO lensing results obtained by Holz \& Wheeler (2002). In Sect. 3 we extend the Holz \& Wheeler (2002) results to Kerr black holes. In Sect. 4 we investigate different geometries among the lens, the source and the observer and discuss the possibility that the next generation high resolution telescopes may have the capabilities necessary to detect such lensing events.

\section{Schwarzschild retro-MACHOs}

The theory of General Relativity predicts that the gravitational field of a massive object deflects light rays of a background source, if the impact parameter is sufficiently close to the massive object. This phenomenon is referred to as gravitational lensing and the massive object causing a detectable deflection is called a gravitational lens.

In gravitational microlensing, photons from a distant source suffer a very small angular deflection and the weak field approximation always works satisfactorily. However, strong field effects may be extremely important if the lens is a collapsed compact object. In fact, very close to a neutron star or a black hole, GR plays an important role and the study of the relativistic images which form (as a consequence of light deflection) allows us to investigate the regions close to the event horizon. The light bending angle in the presence of a static black hole is not limited to small angles but may reach large values ( $\pi$ and odd multiples of $\pi$ if source, observer and retro-MACHO are perfectly aligned, see Holz \& Wheeler 2002) so that photons emerge in the direction of the source itself. Therefore, a Schwarzschild black hole, if illuminated by a powerful light source, shines back with a series of micro-arcsecond rings.

In the case of perfect alignment between the source, the observer and the lens the image of the source, in the plane orthogonal to the line of sight and containing the source, is an annulus with outer and inner radii corresponding to the appropriate impact parameters for photons coming from the top and bottom parts of the source.

However, in the general case the source, the observer and the black hole are not aligned. We consider a reference frame centered at the source $S$ and with axes $X$ and $Y$ on the Earth orbital plane (see Fig. 1). We choose $Y$ to contain the lens in the $Z-Y$ plane and define the misalignment angle $\beta$ as the angle between the observer-lens direction $(\overrightarrow{\mathrm{OL}})$ and the $Y$ axis. Note that the case with $\beta=0$ and Earth in the orbit position $O$ corresponds to perfect alignment.

As the Earth moves on its orbit, the image rings deform into arcs whose angular extent is $\Delta \Theta \simeq 2 \tan ^{-1}\left(R_{\mathrm{S}} / D_{\mathrm{OS}} \sin \theta^{\prime}\right)$, where $\theta^{\prime}$ is the angle between the source-observer and observer-lens $\left(\overrightarrow{\mathrm{SO}^{\prime}}\right.$ and $\left.\overrightarrow{\mathrm{O}^{\prime} \mathrm{L}}\right)$ directions, respectively.

The total area of each image is thus given by (Holz \& Wheeler 2002)

$A_{I} \simeq \pi\left(b_{\mathrm{o}}^{2}-b_{\mathrm{i}}^{2}\right) \frac{\Delta \Theta}{2 \pi}=\left(b_{\mathrm{o}}^{2}-b_{\mathrm{i}}^{2}\right) \tan ^{-1}\left(\frac{R_{\mathrm{S}}}{D_{\mathrm{OS}} \sin \theta^{\prime}}\right)$,

where $b_{\mathrm{o}}$ and $b_{\mathrm{i}}$ represent the outer and inner impact parameters corresponding to bending angles

$\alpha_{\mathrm{d}, \mathrm{B}}=(\pi-\delta) \mp \alpha, \quad \alpha_{\mathrm{d}, \mathrm{S}}=(\pi+\delta) \mp \alpha$

for the bigger and smaller image, respectively. Here $\delta$ is the angle between the $\overrightarrow{\mathrm{SL}}$ and $\overrightarrow{\mathrm{O}^{\prime} \mathrm{L}}$ directions ${ }^{1}$ (i.e. the deflection angle needed for a source photon to reach the observer).

The amplification of each image is given by the ratio between the area of the image given by Eq. (1) to that of the source $A_{\mathrm{S}}=R_{\mathrm{S}}^{2} /\left(4 D_{\mathrm{OS}}^{2}\right)$, i.e.

$\mu_{\mathrm{B}, \mathrm{S}} \simeq\left(b_{\mathrm{o}}^{2}-b_{\mathrm{i}}^{2}\right) \tan ^{-1}\left(\frac{R_{\mathrm{S}}}{D_{\mathrm{OS}} \sin \theta^{\prime}}\right) \frac{D_{\mathrm{OS}}^{2}}{\pi D_{\mathrm{OL}}^{2} R_{\mathrm{S}}^{2}}$.

Finally, setting $b_{\mathrm{o}}$ and $b_{\mathrm{i}}$ to the appropriate impact parameters, the total image amplification is

$\mu=\mu_{\mathrm{B}}+\mu_{\mathrm{S}}$.

Obviously, in the evaluation of the total amplification $\mu$ we need the knowledge of the angles $\theta^{\prime}$ and $\delta$ defined above which, from inspection of Fig. 1, are given by

$\theta^{\prime}(t)=\arccos \left(\frac{\overrightarrow{\mathrm{SO}^{\prime}} \cdot \overrightarrow{\mathrm{O}^{\prime} \mathrm{L}}}{D_{\mathrm{O}^{\prime} \mathrm{S}} D_{\mathrm{O}^{\prime} \mathrm{L}}}\right)$,
$\delta(t)=\arcsin \left(\frac{D_{\mathrm{O}^{\prime} \mathrm{S}}}{D_{\mathrm{O}^{\prime} \mathrm{L}}} \sin (\beta-\gamma)\right)$,

where $\gamma=\arcsin \left(D_{\mathrm{OS}} \sin \beta / D_{\mathrm{SL}}\right)$.

\footnotetext{
${ }^{1}$ Of course, since the observer moves around the Sun on a circular orbit of radius $D_{\mathrm{O}^{\prime} \mathrm{S}} \simeq 1 \mathrm{AU}$ with angular velocity $\omega_{\mathrm{E}} \simeq 2 \pi / T_{\mathrm{E}}$ (being $T_{\mathrm{E}}$ the orbital period), the Earth position angle $\phi$ and the angles $\delta$ and $\theta^{\prime}$ (see Fig. 1) are time dependent.
} 


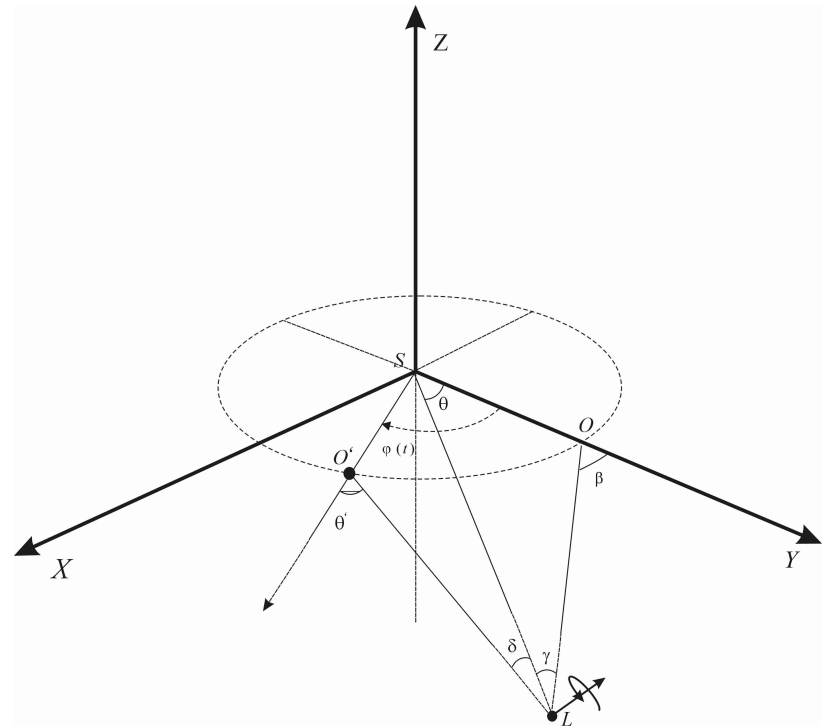

Fig. 1. The geometry of a retro-lensing event is shown in the case of a general configuration for the lens, source and observer positions. Photons emitted by the Sun S, in the center of the reference frame, move towards the retro-MACHO L (a Schwarzschild or a Kerr black hole) which, for simplicity, is placed on the $Z-Y$ plane. Light rays, which interact with the black hole strong gravitational field, may suffer a path deflection to bending angle multiples of $\pi$ and reach the observer $\mathrm{O}^{\prime}$. The arrow on the lens represents the black hole spin vector which lies perpendicular to the lens-observer-source plane.

At this stage, we only need to evaluate the photon impact parameter values $b_{\mathrm{o}}$ and $b_{\mathrm{i}}$ so that light rays coming from the source are deflected toward the observer. For a Schwarzschild black hole with mass $M$, Chandrasekhar (1983) has found that, in the case of large deflection angle $\alpha_{\mathrm{d}}$, the photon impact parameter, in geometrical units $G=c=1$, is given by

$b\left(\alpha_{\mathrm{d}}\right)=b_{1, \mathrm{~S}}+b_{2, \mathrm{~S}} \mathrm{e}^{-\alpha_{\mathrm{d}}}$,

where

$b_{1, \mathrm{~S}}=3 \sqrt{3} M$

$b_{2, \mathrm{~S}}=648 \sqrt{3} \mathrm{e}^{-\pi} M(\sqrt{3}-1)^{2} /(\sqrt{3}+1)^{2}$.

Consequently, for each position of the Earth around the Sun it is possible to evaluate the angles $\theta^{\prime}(t)$ and $\delta(t)$, the bending angles $\alpha_{\mathrm{d}, \mathrm{B}}$ and $\alpha_{\mathrm{d}, \mathrm{S}}$ and, through Eqs. (3) and (6), the total image amplification (4) as seen by the observer.

The expected light curves (i.e. magnitudes $m$ corresponding to a certain amplification $\mu$ as a function of time) for a retrolensing event due to a Schwarzschild black hole are shown in Fig. 2 for different misalignment angles $\beta$.

\section{Kerr retro-MACHOs}

The results by Holz \& Wheeler (2002) on retro-lensing events hold in the case of Schwarzschild black holes. However, in general, a black hole is characterized by a non-zero intrinsic angular momentum which breaks the spherical symmetry and affects the gravitational field around the compact object.

Indeed, black holes are the ultimate stage of stellar evolution and may form by SNII explosions. Since stars rotate,

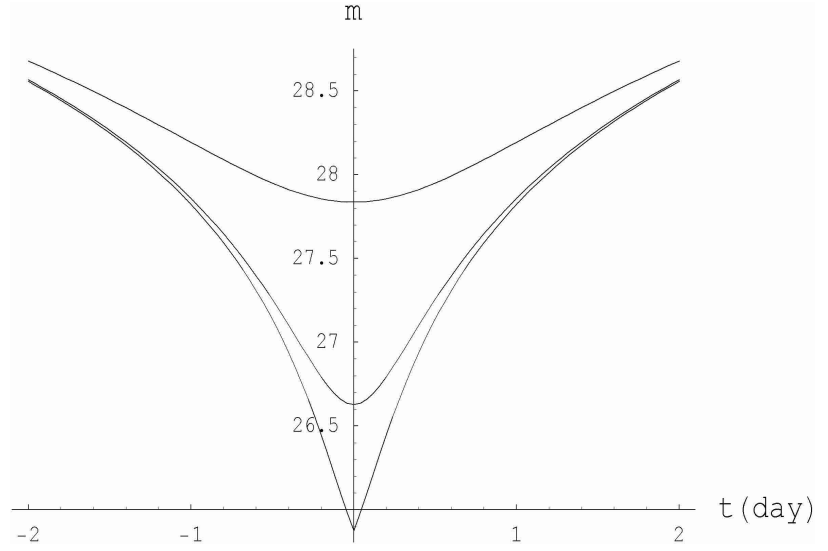

Fig. 2. The expected visual magnitude $m$ of the arcs forming close to a $10 M_{\odot}$ Schwarzschild black hole (with $a=0$ at $0.01 \mathrm{pc}$ from the Earth) as a function of time (i.e of the Earth position) is shown. The curves correspond to different displacement angles $\beta$. In particular, from top to bottom, we set $\beta=1^{\circ}, \beta=R_{\odot} / 1 \mathrm{AU}$ and $\beta=0^{\circ}$, respectively. Note that $t=0$ means, referring to Fig. 1, that the Earth is on the $Y$ axis.

angular momentum conservation implies that black holes also rotate. Black hole rotation is also confirmed by recent $X$-ray observations of Fe line width (Zakharov et al. 2002). Moreover, super massive black holes at the center of QSOs, AGNs and galaxies show beamed jet emission implying that they have non zero angular momentum.

Thus, due to black hole angular momentum $J$, we expect a modification in the phenomenology of both the usual gravitational lensing and the retro-MACHO microlensing. Such modifications are evaluated by a second order expansion of the photon deflection angles in the spin parameter $a=J /\left(M c R_{\mathrm{Sch}}\right)$. Here, $R_{\text {Sch }}=2 G M / c^{2}$ is the black hole Schwarzschild radius $^{2}$. We consider light trajectories on the black hole equatorial plane and a more general treatment will be addressed elsewhere (Zakharov et al. 2003). However, we expect that the maximal effect due to the black hole spin occurs for photons lying in the equatorial plane while for partially inclined orientations the effect becomes closer to the Schwarzschild case (that is recovered for $\theta=0$ ).

A simple and reliable method to investigate the subject has been recently proposed by Bozza (2003) (for an exhaustive review of the topics see also Bozza 2002a) who revisited the Schwarzschild and Kerr black hole lensing in a strong gravitational field.

Considering light rays on the black hole equatorial plane $(\theta=\pi / 2)$, the Kerr line element in Boyer-Lindquist coordinates $(t, x, \theta, \phi)$ is given by

$\mathrm{d} s^{2}=A(x) \mathrm{d} t^{2}-B(x) \mathrm{d} x^{2}-C(x) \mathrm{d} \phi^{2}+D(x) \mathrm{d} t \mathrm{~d} \phi$,

${ }^{2}$ Note that the definition we use for the rotation parameter $a$ corresponds to half of the value in the usual notation $a=2 J /\left(M c R_{\text {Sch }}\right)$. Indeed, following Bozza (2003) we measure distances in units of the Schwarzschild radius and not in units of the gravitational radius as is usually done (see e.g. Shapiro \& Teukolsky 1983). 
with

$$
\begin{aligned}
& A(x)=1-\frac{1}{x} \\
& B(x)=\left(1-\frac{1}{x}+\frac{a^{2}}{x^{2}}\right)^{-1} \\
& C(x)=x^{2}+a^{2}+\frac{a^{2}}{x} \\
& D(x)=2 \frac{a}{x},
\end{aligned}
$$

where $x$ is the distance from the spinning black hole measured in units of the Schwarzschild radius in natural units $R_{\mathrm{Sch}}=2 M$ and $a=J /\left(2 M^{2}\right)$.

Following Bozza (2003) the impact parameter $b$ of light rays approaching the black hole is

$b=\frac{-D_{0}+\sqrt{4 A_{0} C_{0}+D_{0}^{2}}}{2 A_{0}}$.

Here and in the following all the metric functions with the subscript 0 are evaluated at $x=x_{0}$ which is the photon minimum distance from the black hole.

After a straightforward calculation, the whole deflection angle (i.e. the bending angle for a photon with impact parameter $b$ ) is given by

$\alpha_{\mathrm{d}}=2 \int_{x_{0}}^{\infty} \frac{\mathrm{d} \phi}{\mathrm{d} x} \mathrm{~d} x-\pi$,

where

$\frac{\mathrm{d} \phi}{\mathrm{d} x}=P_{1}\left(x, x_{0}\right) P_{2}\left(x, x_{0}\right)$,

with

$P_{1}\left(x, x_{0}\right)=\frac{\sqrt{B}\left(2 A_{0} A b+A_{0} D\right)}{\sqrt{C A_{0}} \sqrt{4 A C+D^{2}}}$

$P_{2}\left(x, x_{0}\right)=\left(A_{0}-A \frac{C_{0}}{C}+\frac{b}{C}\left(A D_{0}-A_{0} D\right)\right)^{-1 / 2}$.

The deflection angle $\alpha_{\mathrm{d}}$ grows as $x_{0}$ decreases diverging at the radius of the photosphere $x_{m}$ (to be defined below). In this case the photon is captured by the black hole. Defining the new variable $z=\left(x-x_{0}\right) / x$ and hence $x=x_{0} /(1-z)$, the integral in Eq. (11) becomes

$\alpha_{\mathrm{d}}=\int_{0}^{1} R\left(z, x_{0}\right) f\left(z, x_{0}\right) \mathrm{d} z-\pi$,

with

$R\left(z, x_{0}\right)=2 \frac{\left(1-y_{0}\right)}{A^{\prime}(x)} P_{1}\left(x, x_{0}\right)$

$f\left(z, x_{0}\right)=P_{2}\left(x, x_{0}\right)$.

The function $R\left(z, x_{0}\right)$ is regular for any values of $z$ while the function $f\left(z, x_{0}\right)$ diverges as $z \rightarrow 0$. To find the divergence order, the argument of the square root in $f\left(z, x_{0}\right)$ may be expanded in powers of $z$ obtaining (Bozza 2003)

$f_{0}\left(z, x_{0}\right)=\frac{1}{\sqrt{c_{1}\left(x_{0}, a\right) z+c_{2}\left(x_{0}, a\right) z^{2}}}$,

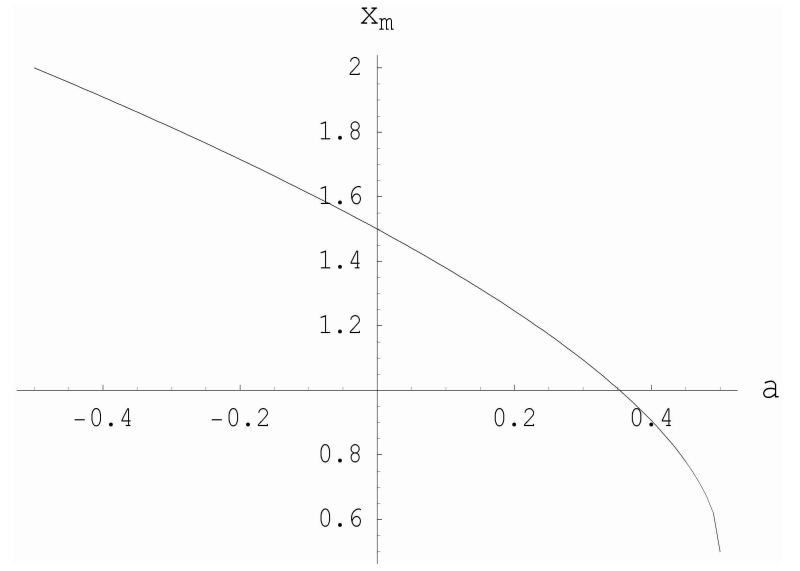

Fig. 3. The radius of the photosphere as a function of the black hole spin parameter $a$ is shown. As expected, for positive values of $a$ (i.e. for light rays co-rotating with the black hole) photons are allowed to get closer to the black hole. For $a \rightarrow 0$ the photosphere radius approaches the last stable orbit radius value for a Schwarzschild black hole $\left(x_{m}=3 / 2\right)$.

where $c_{1}\left(x_{0}, a\right)$ and $c_{2}\left(x_{0}, a\right)$ represent the first and second order coefficients of the expansion. Therefore, when $c_{1}\left(x_{0}, a\right)$ is different from zero the leading order term in the integrand is $\propto z^{-1 / 2}$, so that the integral in Eq. (14) converges. Instead, the integral diverges in the case $c_{1}\left(x_{0}, a\right)=0$, corresponding to photons being captured by the black hole. Accordingly, the photosphere radius $x_{m}$ is defined as the outermost solution of equation $c_{1}\left(x_{0}, a\right)=0$. This condition is equivalent to $8 a^{2}-x_{0}\left(3-2 x_{0}\right)^{2}=0$ (for details see Bozza 2003). Of the three solutions of the cubic equation above, one is irrelevant as it lies inside the horizon while the other two correspond to the co-rotating and counter-rotating photons. The counter-rotating case may also be obtained by taking $a<0$. The roots are displayed in Fig. 3.

Once the photosphere radius $x_{m}$ (for a given value of $a$ ) is known, one can evaluate the deflection angle $\alpha_{d}$ from Eq. (14). Thus, retaining only terms up to $O\left(x_{0}-x_{m}\right)$, one obtains

$\alpha_{\mathrm{d}}\left(x_{0}\right)=-k_{1} \ln \left(\frac{x_{0}}{x_{m}}-1\right)+k_{2}+O\left(x_{0}-x_{m}\right)$,

where

$k_{1}=\frac{R\left(0, x_{m}\right)}{\sqrt{c_{2}\left(x_{m}, a\right)}}$

$k_{2}=-\pi+k_{1} \ln \frac{2\left(1-y_{m}\right)}{A^{\prime}\left(x_{m}\right) x_{m}}+I_{R}$

$I_{R}=\int_{0}^{1}\left[R\left(z, x_{m}\right) f\left(z, x_{m}\right)-R\left(0, x_{m}\right) f_{0}\left(z, x_{m}\right)\right] \mathrm{d} z$.

The impact parameter $b$ in Eq. (10) can now be expanded in powers of $\left(x_{0}-x_{m}\right)$ up to the second order term (the first order coefficient vanishes) obtaining

$b \simeq b_{0}\left(x_{m}\right)+b_{2}\left(x_{m}\right)\left(x_{0}-x_{m}\right)^{2}$. 

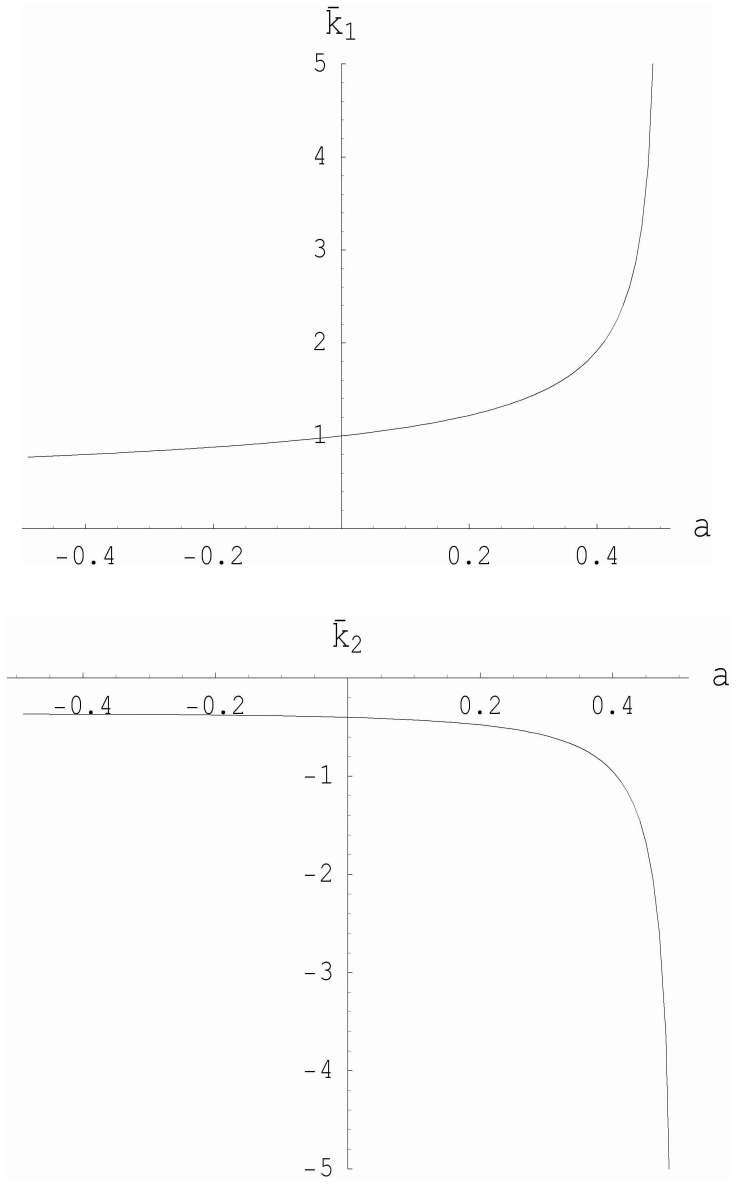

Fig. 4. The coefficients $\bar{k}_{1}$ (upper panel) and $\bar{k}_{2}$ (bottom panel) appearing in Eq. (20) are plotted as functions of the black hole spin parameter a. For details see text.

Eliminating the closest approach distance $x_{0}$ in Eqs. (17) and (19) and rearranging the terms one finally gets (Bozza 2003)

$\alpha_{\mathrm{d}}(b)=-\bar{k}_{1} \ln \left(\frac{b}{b_{0}\left(x_{m}\right)}-1\right)+\bar{k}_{2}+O\left(b-b_{0}\left(x_{m}\right)\right)$,

with

$\bar{k}_{1}=\frac{k_{1}}{2}$,

$\bar{k}_{2}=k_{2}+\frac{k_{1}}{2} \ln \frac{b_{2}\left(x_{m}\right) x_{m}^{2}}{b_{0}\left(x_{m}\right)}$.

In Fig. 4 we plot the coefficients $\bar{k}_{1}$ and $\bar{k}_{2}$ as a function of the black hole spin parameter. We note that these coefficients diverge for $a \rightarrow 0.5$ implying that Eq. (20) can be used only for low enough values of $a$ for which $x_{0}-x_{m} \ll 1$ holds.

Therefore, by solving Eq. (20) with respect to the impact parameter $b$, one finally obtains the generalization of the Chandrasekhar relation for Schwarzschild black holes given in Eq. (6) to Kerr black holes

$b\left(\alpha_{\mathrm{d}}\right) \simeq b_{1, \mathrm{~K}}+b_{2, \mathrm{~K}} \mathrm{e}^{-\alpha_{\mathrm{d}} / \bar{k}_{1}}$,

with

$b_{1, \mathrm{~K}}=2 b_{0}\left(x_{m}\right) M, \quad b_{2, \mathrm{~K}}=2 b_{0}\left(x_{m}\right) M \mathrm{e}^{-\bar{k}_{2} / \bar{k}_{1}}$.

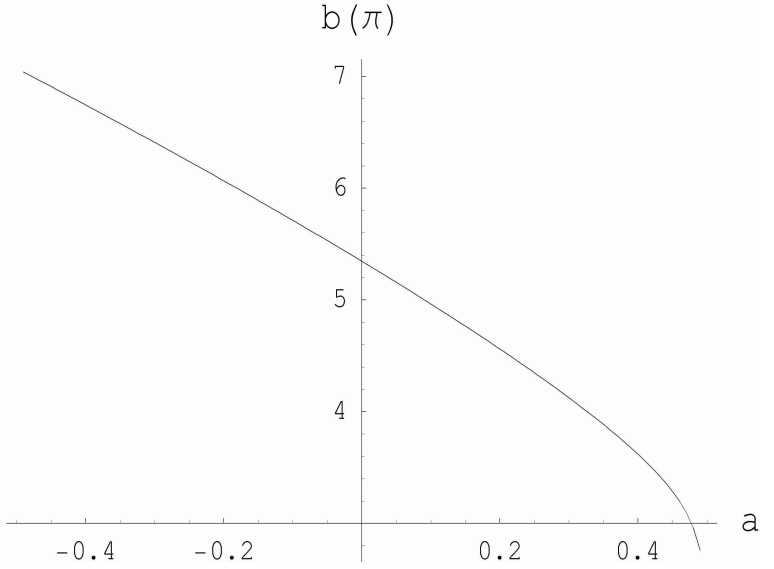

Fig. 5. The impact parameter $b$, evaluated for the deflection angle $\alpha_{\mathrm{d}}=$ $\pi$, is shown as a function of the black hole spin parameter $a$. Note that for $a \rightarrow 0$ the impact parameter approaches the result $b=5.34664$ for a Schwarzschild black hole given by Chandrasekhar (1983).

Note that the factor 2 in the previous relations (23) has been introduced to make our expression comparable with that in Eq. (6). It is also easy to verify that for $a \rightarrow 0$, Eq. (22) reduces to the result (6) given by Chandrasekhar (1983) for Schwarzschild black holes.

We are now ready to apply this formalism to the retrolensing phenomenon. In Fig. 5, assuming $\alpha_{d}=\pi$, we plot the impact parameter $b(\pi)$ as a function of the black hole spin parameter $a$. As one can see, for co-rotating photons $(a>0)$ the impact parameters decreases with respect to the Schwarzschild case (i.e. photons may get closer to the black hole).

Let us now consider the Sun as the light source and a black hole of mass $10 M_{\odot}$ at a distance of $0.01 \mathrm{pc}$. Since the solar luminosity in the optical band is $L_{\odot} \simeq 4 \times 10^{33} \mathrm{erg} \mathrm{s}^{-1}$, corresponding to an optical visual magnitude $m_{\odot}=-26.8$, the expected magnitude $m$ of the retro-lensed image can be evaluated as

$m=m_{\odot}-2.5 \log \mu$,

$\mu$ being the total amplification given by Eq. (4). In the case of a Schwarzschild black hole with $a=0$, the expected apparent magnitude is given in Fig. 2 as a function of the time $t$ (corresponding to the Earth position on its orbit), for different values of the misalignment angle $\beta$ (see also Holz \& Wheleer 2002).

In the case of a Kerr black hole we can evaluate the Sun image amplification by using Eqs. (3)-(5), in the most favorable case of perfect alignment $(\beta=0)$. For this purpose we only need to evaluate, through Eq. (22), the impact parameters corresponding to the bending angles for which light rays emitted by the Sun may reach the observer after deflection of the angles $\alpha_{\mathrm{d}, \mathrm{B}}$ and $\alpha_{\mathrm{d}, \mathrm{S}}$ defined in Sect. 2. For a $10 M_{\odot}$ Kerr black hole with spin parameter $a=0.1$ at a distance of $0.01 \mathrm{pc}$ and misalignment angle $\beta=0$, the expected retro-lensing light curve is given in Fig. 6 (red solid line). In the same figure we also give the light curve (dashed red line) corresponding to counter-rotating photons. As expected, for a Kerr black hole with spin parameter $a$, only a modification up to the second order in the retro-MACHO amplification appears, as is evident 


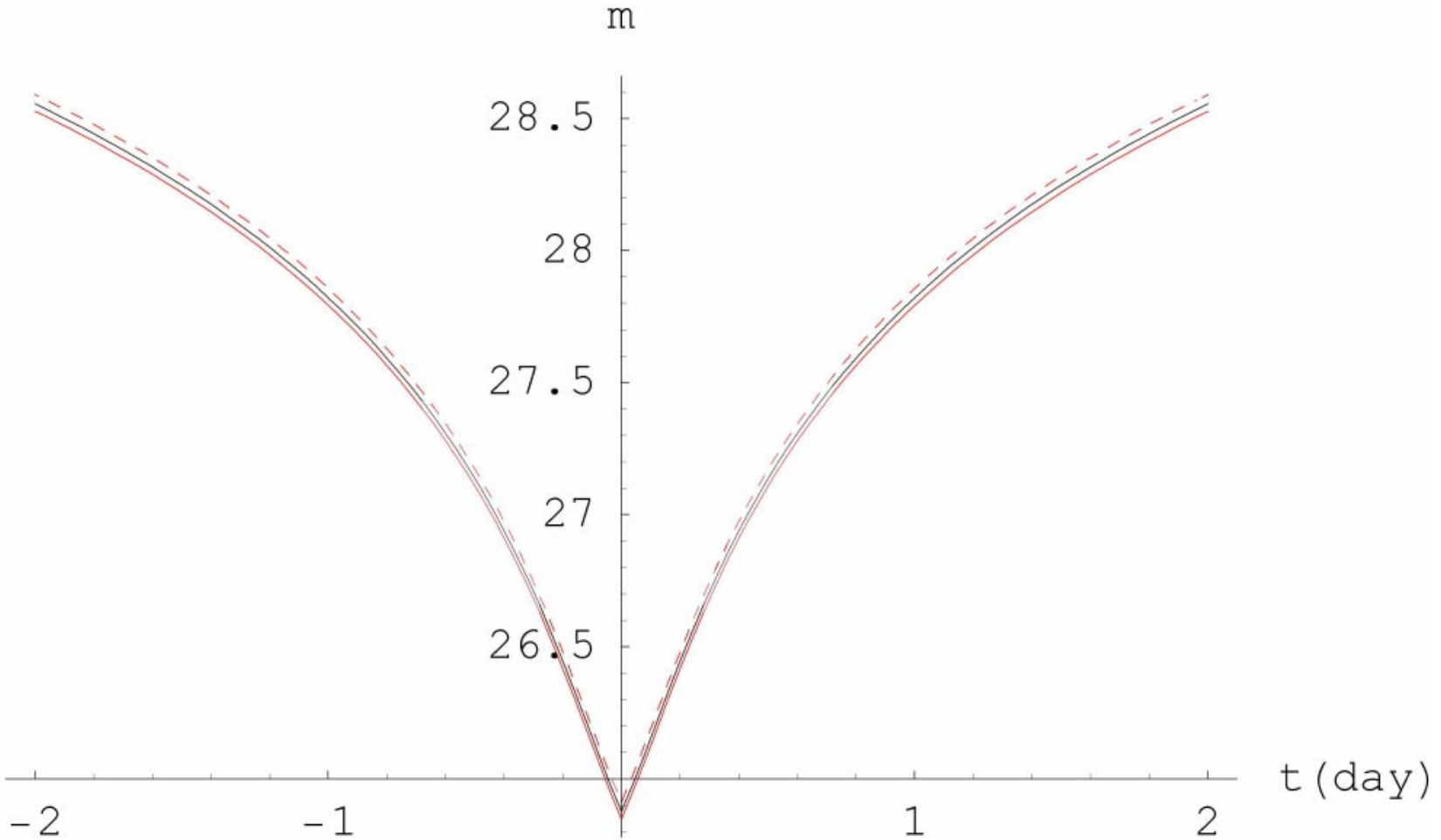

Fig. 6. The expected retro-lensing light curves for a Kerr black hole with mass $10 M_{\odot}$ and spin parameter $a=0.1$ is given as a function of the time (i.e. of the Earth position in its orbit). The solid red line corresponds to the magnitude of the image formed by co-rotating photons while the dashed red line is the magnitude of the image formed by counter-rotating photons. The central black line corresponds to a retro-lensing event involving a Schwarzschild black hole with the same mass. The light source is the Sun and the black hole is placed at 0.01 pc from Earth.

by inspecting Fig. 6 where the lightcurves for the Kerr black hole case are reported (dashed and solid red lines) together with the corresponding one for a Schwarzschild black hole with the same mass and $\beta=0$ (central black solid line $)^{3}$. We also notice that we have neglected multiple rotations of the photons around the black hole. As emphasized by Holz \& Wheeler (2002), photons may form a series of deformed rings corresponding to deflection angles that differ by $2 n \pi$ (with $n$ as integer number). However, it is clear that the brightest image is that corresponding to the outermost ring (obtained for $\alpha_{\mathrm{d}} \simeq \pi$ ) so that neglecting the other images does not change substantially the retrolensing image brightness.

\section{Discussion}

It has been shown by Holz \& Wheeler (2002) that the limiting distance $D_{\mathrm{L}}$ at which the rings around a black hole of mass $M$ illuminated by our Sun can be observed is given by

$D_{\mathrm{L}}=0.02 \mathrm{pc} \times\left[10^{(m-30) / 2.5}\left(M / 10 M_{\odot}\right)^{2}\right]^{1 / 3}$,

where the ring magnitude of the retro-lensing event has been chosen to be 30 (see Fig. 2). For example, if the Sun is the

\footnotetext{
${ }^{3}$ Co-rotating and counter-rotating photons should produce an image scintillation due to their relative time delay. However, the time scale for such scintillations goes from $\sim 10^{-5} \mathrm{~s}$ to $\sim 10 \mathrm{~s}$ for black holes with mass in the range $1 M_{\odot}-10^{6} M_{\odot}$. Due to the short scintillation time scale with respect to the integration time required by observation, the net result is simply that of seeing the average, which coincides with the light curve for the Schwarzschild black hole.
}

light source and the black hole has mass $10 M_{\odot}$, the limiting distance at which the retro MACHO can be detected is of only $0.02 \mathrm{pc}$ for an instrument with $m=30$ (such as HST). On the other hand, it is very unlikely that black holes so close to the Earth exist. However, the most convenient geometry to get luminous rings around a black hole would be that of a very bright star close to a black hole at some distance $D_{\mathrm{L}}$ from Earth ${ }^{4}$. In this case, and for the most general geometrical configuration of source, lens and observer with relative distances $D_{\mathrm{OL}}, D_{\mathrm{LS}}$ and $D_{\mathrm{OS}}$, the instrumental limiting magnitude $\bar{m}$ necessary to see the rings is thus given by

$\bar{m} \geq m_{\lambda}-2.5 \log \mu$,

$m_{\lambda}$ being the magnitude of the source in a certain electromagnetic band and $\mu$ the ring amplification given by Eq. (4). In the particular case of perfect alignment between observer, lens and source, the previous relation reads

$\bar{m} \geq m_{\lambda}-2.5 \log \left[1.04\left(\frac{M}{M_{\odot}}\right)^{2}\left(\frac{R_{\odot}}{R_{\mathrm{S}}}\right) \frac{D_{\mathrm{OS}}^{2}}{D_{\mathrm{LS}} D_{\mathrm{OL}}^{2}}\right]$,

\footnotetext{
${ }^{4}$ It has been shown that the best chance of observing retro-lensing images in the future is by looking towards the galactic center black hole in Sgr $\mathrm{A}^{*}$ around which a very bright star (named S2) with a mass of about $15 M_{\odot}$ is orbiting. The resulting magnitude of the retrolensing images in the Schwarzschild case are in the range 33.3-36.8 (depending on the star distance from the black hole) in the $K$-band, close to the limiting magnitude of the next generation of space-based telescopes (De Paolis et al. 2003a).
} 
which reduces to Eq. (25) taking the geometry adopted by Holz $\&$ Wheeler (2002). Notice that in the previous equation we are considering all the distances $\left(D_{\mathrm{OS}}, D_{\mathrm{LS}}\right.$ and $\left.D_{\mathrm{OL}}\right)$ measured in $\mathrm{cm}$.

As is clear from Fig. 6, the spin effect on the image brightness is a minor effect and so it cannot increase substantially the image amplification with respect to the Schwarzschild black hole case. Moreover, as already stated, our analysis is confined to photons lying in the black hole equatorial plane and the retro-lensing image magnitude is calculated by using Eq. (4). This equation is rigorously valid only in the Schwarzschild case since it assumes that each part of the image is isoluminous (and this is not true for Kerr black holes). However, if we limit the estimate to only the total magnitude of the retro-lensing images, we do not expect a substantial change with respect to the Schwarzschild case since the increase in the luminosity of the corotating edge should approximately compensate the luminosity decrease of the counter-rotating edge.

We emphasize again that our treatment is valid only for slowly rotating black holes but it is clear that increasing the rotation parameter $a$, the brightness of the retro-lensing images also increases. A complete (purely numerical) treatment of this issue - valid for arbitrarily fast rotating black holes - will be published elsewhere (De Paolis et al. 2003b).

Since in the slow rotation limit the light curves are almost indistinguishable from the Schwarzschild case, the most important effect of the black hole spin is that of deforming the ring shape with respect to the Schwarzschild case (Peter 1976; Bray 1986). A detailed analysis of this effect will be presented elsewhere (Zakharov et al. 2003). Detecting the shape of the ring images might give a unique possibility of investigating the black hole parameters (spin $a$, mass $M$ and relative position of observer-lens-black hole) in an active way.

To really observe the ring shape, the detecting instrument must have a high enough angular resolution in the range 1-10 $\mu$ arcsec for most cases of interest. Future space-based instruments like the Space Interferometry Mission (SIM) (see Shao 1998 for technical details), GAIA Satellite (see e.g. Belokurov \& Evans 2002), FAME (Johnston et al. 2000) and MAXIM (2003) would have the angular resolution capabilities to resolve such images and therefore will be able to observe
retro-MACHOs if the instrumental limiting magnitude satisfies Eq. (26).

\section{References}

Agol, E., Kamionkowski, M., Koopmans, L. U. E., \& Blandford, R. 2002, ApJ, 576, L131

Alcock, C., Akerloff, C. W., Allsman, R. A., et al. 1993, Nature, 365, 621

Alcock, C., Allsman, R. A., Alves, D. R., et al. 2000, ApJ, 542, 281

Aubourg, E., Bareyre, P., Brehin, S., et al. 1993, Nature, 365, 623

Belokurov, V. A., \& Evans, N. W. 2002, MNRAS, 331, 649

Bennett, D. P., Becker, A. C., Quinn, J. L., et al. 2002, ApJ, 579, 639

Bozza, V. 2002, Phys. Rev. D, 66, 10

Bozza, V. 2003, Phys. Rev. D, 67, 103006

Bray, I. 1986, Phys. Rev. D, 34, 367

Canizares, C. R., 1982, ApJ, 263, 508

Chandrasekhar, S. 1983, Mathematical Theory of Black Holes (Oxford: Clarendon Press)

De Paolis, F., Geralico, A., Ingrosso, G., \& Nucita, A. A. 2003, A\&A, 409, 809

De Paolis, F., Geralico, A., Ingrosso, G., Nucita, A. A., \& Qadir, A. 2003, in preparation

Hewitt, J. N., Turner, E. L., Schneider, D. P., et al. 1988, Nature, 333, 537

Holz, D. E., \& Wheeler, J. A. 2002, ApJ, 57, 330

Johnston, K., Gaume, R., Harris, F., et al. 2000, AAS, 197, 1403

Lynds, R., \& Petrosian, V. 1989, ApJ, 336, 1

Mao, S, Smith, M. C., Wozniak, P., et al. 2002, MNRAS, 329, 349

MAXIM web page at: http://maxim.gsfc.nasa.gov/

Paczyński, B. 1986, ApJ, 304, 1

Peter, J. Y. 1976, Phys. Rev. D, 14, 3281

Quinn, J. L., Alcock, C., Allsman, R. A., et al. 1999, AAS, 31, 1422

Schneider, P., Ehlers, J., \& Falco, E. E. 1992, Gravitational Lenses (Cambridge: Springer-Verlag)

Shao, M. 1998, Astronomical Interferometry, ed. R. D. Reasenberg, Proc. SPIE, 3350, 536

Shapiro, S. L., \& Teukolsky, S. A. 1983, Black Holes, White Dwarfs, and Neutron Stars (New York: John Wiley \& Sons)

Soucail, G., Fort, B., Mellier, Y., \& Picat, J. P. 1987, A\&A, 172, 14

Stanek, K. Z., Paczynski, B., Udalski, A., et al. 1995, AAS, 186, 304

Zakharov, A. F., Kardashev, N. S., Lukash, V. N., \& Repin, S. V. 2002, MNRAS, 342, 1325

Zakharov, A. F., Nucita, A. A., Geralico, A., et al. 2003, in preparation 